\title{
Geometric Conditions for Regularity of Viscosity Solution to the Simplest Hamilton-Jacobi Equation ${ }^{\star}$
}

\author{
Vladimir V. Goncharov ${ }^{\star \star}$ and Fátima F. Pereira \\ Universidade de Évora, CIMA-UE, Rua Romão Ramalho 59, \\ 7000-671, Évora, Portugal \\ \{goncha,fmfp\}@uevora.pt
}

\begin{abstract}
Continuing research in [13] and 14] on well-posedness of the optimal time control problem with a constant convex dynamics in a Hilbert space we adapt one of the regularity conditions obtained there to a slightly more general problem, where nonaffine additive term appears. We prove existence and uniqueness of a minimizer in this problem as well as continuous differentiability of the value function, which can be seen as the viscosity solution to a Hamilton-Jacobi equation, near the boundary.
\end{abstract}

Keywords: optimal time control problem, viscosity solution, eikonal equation, duality mapping, proximal normals, proximal regularity, Hölder continuity.

\section{Introduction}

Let us start with the first order partial differential equation in finite dimensions

$$
\Gamma(x, u(x), \nabla u(x))=0
$$

where $\Gamma: \Omega \times \mathbb{R} \times \mathbb{R}^{n} \rightarrow \mathbb{R}$ is a continuous function, nonlinear with respect to (w.r.t.) the third variable; $\Omega \subset \mathbb{R}^{n}$ is an open bounded region. Due to applications in optimal control and dynamical systems (1) is traditionally called (stationary) Hamilton-Jacobi equation. There are various notions of solutions to this equation. For instance, a function $u: \bar{\Omega} \rightarrow \mathbb{R}$ of class $C(\bar{\Omega}) \cap C^{1}(\Omega)$ satisfying (11) for all $x \in \Omega$ is said to be classical solution, while a Lipschitz continuous function $u: \bar{\Omega} \rightarrow \mathbb{R}$ such that (1) holds for almost each (a.e.) $x \in \Omega$ is usually called generalized (or almost everywhere) solution. Speaking about solutions of (11) we always have in mind some prescribed boundary condition

$$
u(x)=\theta(x), \quad x \in \partial \Omega,
$$

\footnotetext{
* Work is realized in framework of the project "Variational Analysis: Theory and Applications" (PTDC/MAT/111809/2009) financially supported by Fundação para Ciência e Tecnologia (FCT), the Portuguese institutions COMPETE, QREN and the European Regional Development Fund (FEDER).

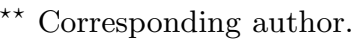

D. Hömberg and F. Tröltzsch (Eds.): CSMO 2011, IFIP AICT 391, pp. 245 254, 2013.

(C) IFIP International Federation for Information Processing 2013
} 
where $\theta: \bar{\Omega} \rightarrow \mathbb{R}$ is a (continuous) given function. Since in practice a classical solution to the boundary value problem (11)-(2) often fails to exist while generalized solution may not be unique, another physically reasonable concept (so named viscosity solution) was introduced by M. Crandall and P.-L. Lions in 1983 (see 6]) while similar constructions under different names were known earlier (see, e.g., [16], [12], [15]). This concept was mainly based on the idea of "vanishing viscosity" in the sense that each viscosity solution is the uniform limit of the sequence of solutions $u^{\varepsilon}(\cdot)$ to the respective boundary value problems for the nonlinear elliptic equations

$$
\Gamma(x, u, \nabla u)-\varepsilon \Delta u=0
$$

as $\varepsilon \rightarrow 0+$ where $\Delta=\sum_{i=1}^{n} \frac{\partial^{2}}{\partial x_{i}^{2}}$ is the Laplace operator (notice that (3) has a unique classical solution for each $\varepsilon>0$ small enough due to Theorem 3.2 [15]). The exact definition of viscosity solution can be given either in terms of the suitable test functions (similarly as the notion of the generalized solutions of linear PDE in the sense of distributions), or by involving a Fréchet generalization of the gradient of a function at the point of nondifferentiability. It turned out that for each suitable boundary data $\theta(\cdot)$ a (continuous) viscosity solution to the problem (1)-(2) exists, is unique and stable w.r.t. both $\theta(\cdot)$ and $\Gamma(\cdot)$. Furthermore, it is agreed with other types of solutions. In particular, each viscosity solution belonging to $C^{1}(\Omega)$ is classical. For the main results of Theory of Viscosity Solutions, very developed and powerful field of the modern mathematics, we refer to [1]- 2] and the bibliography therein. For a concise survey of viscosity solutions in finite dimensions see also the excellent tutorial lessons by A. Bressan [3].

Afterwards, the concept and the main results concerning viscosity solutions were generalized to Banach spaces with the Radon-Nikodym property (see [7], [8]), in particular, to Hilbert spaces. The gradient $\nabla u$ in (11) is understood then in the sense of Fréchet. Notice that although the definition based on the Fréchet sub- and superdifferentials remains the same, the interpretation of viscosity solutions via "vanishing viscosity" is no longer valid in infinite dimensions. The motivation, however, comes now from the Theory of Differential Games.

In our paper we deal only with the case when the hamiltonian $\Gamma$ in (1) does not depend of $x$ neither $u$, and is convex w.r.t. the third variable. Already S. N. Kružkov studied in [15] such Hamilton-Jacobi equations arizing from the geometric optics. For instance, when $n=3$ and $\Gamma(x, u, \xi)=|\xi|-a$, with a constant $a>0$, one has the so called eikonal equation describing the propagation of a light wave from a point source placed at the origin in homogeneous medium with refraction index $1 / a$. If, instead, this medium is anisotropic and has constant coefficients of refraction of light rays parallel to the coordinate axes ( $\operatorname{say} c_{i}$ ) then the propagation of light can be described by the (more general) elliptic equation

$$
\sum_{i=1}^{n} c_{i}^{2} u_{x_{i}}^{2}-1=0
$$


If, besides that, the medium moves with a constant velocity $\vec{v}$ then the equation contains already a linear additive term and admits the form

$$
\sum_{i=1}^{n} c_{i}^{2} u_{x_{i}}^{2}+\frac{2}{c}\langle\vec{v}, \nabla u\rangle-1=0
$$

where $c$ means the speed of the light in a vacuum.

In general, denoting by $F$ the closed convex hull of the set of zeros

$$
\left\{\xi \in \mathbb{R}^{n}: \Gamma(\xi)=0\right\}
$$

and assuming $F$ to be bounded with $\operatorname{int} F \neq \emptyset$ (the hamiltonians in (44) and (5) satisfy these conditions), the equation (11) can be reduced to

$$
\rho_{F}(\nabla u(x))-1=0,
$$

where $\rho_{F}(\cdot)$ is the Minkowski functional (gauge function) associated to $F$,

$$
\rho_{F}(\xi):=\inf \{\lambda>0: \xi \in \lambda F\} .
$$

More precisely, it was proved in [4 that under appropriate conditions involving a kind of geometric compatibility of $F, \theta(\cdot)$ and the domain $\Omega$ the (unique) viscosity solution $\hat{u}(\cdot),\left.\hat{u}\right|_{\partial \Omega}=\theta$, of (6) is the viscosity solution of the problem (1)-(2) (belonging to the space $W^{1, \infty}(\Omega)$ ) and vice versa. Furthermore, this viscosity solution can be given by the formula

$$
\hat{u}(x)=\inf _{y \in C}\left\{\rho_{F^{0}}(x-y)+\theta(y)\right\}
$$

whenever $\theta: \mathbb{R}^{n} \rightarrow \mathbb{R}$ is a Lipschitz continuous function such that

$$
\nabla \theta(x) \in \operatorname{int} F \text { for a.e. } x \in \mathbb{R}^{n} \text {. }
$$

Here $C:=\mathbb{R}^{n} \backslash \Omega$ and $F^{0}$ is the polar set for $F$.

Let now $H$ be a Hilbert space with the norm $\|\cdot\|$ and the inner product $\langle\cdot, \cdot\rangle$. Then the convolution (77) remains the unique viscosity solution to the equation (6) with the boundary data $u(x)=\theta(x), x \in C$, whenever the slope condition

$$
\theta(x)-\theta(y)<\rho_{F^{0}}(x-y) \quad \forall x, y \in C
$$

holds. Notice that the inequality (9) follows from (8) in finite dimensions while in an arbitrary Hilbert space from the inclusion $\partial^{c} \theta(x) \subset F, x \in H$, where $\partial^{c}$ is the Clarke generalized gradient of a Lipschitz continuous function.

So, we are interested in regularity properties of the function (7), which were well studied when $\theta \equiv 0$ (see [10], [13], [14]). In the latter case let us notice the following:

1) existence and regularity of the (Fréchet) gradient $\nabla \hat{u}(x)$ depends on uniqueness (in infinite dimensions also on existence) of a minimizer in (7); 
2 ) it is not possible that $\nabla \hat{u}(x)$ exists everywhere out of $C$ unless some special situations;

3 ) the function $\hat{u}(x)$ can be interpreted as the minimal time necessary to achieve the closed set $C$ from $x \in H \backslash C$ by trajectories of the differential inclusion $\dot{x}(t) \in-F^{0}$.

Taking into account 1) and 2) it is natural to study the regularity only in an (open) neighbourhood of $C$ (target set due to 3 ). If $F=\bar{B}$ is the closed unit ball centred in the origin then $\hat{u}(\cdot)$ is nothing else than the distance from $C$, and the minimizers in (7) are the usual metric projections onto $C$. In this case the (necessary and sufficient) condition guaranteeing well-posedness of the problem and the (Lipschitz) continuity of the gradient $\nabla \hat{u}(x)$ near $C$ is so named $\varphi$ convexity (or proximal smoothness) of the set $C$ well studied up to now (see survey [9] and the bibliography therein).

As concerns an arbitrary gauge $F$ (and $\theta \equiv 0$ ) then in [13, 14 two different hypotheses are given, under which both a unique minimizer in (7) (that is a point on the boundary $\partial C$ attained from $x$ for the minimal time) and the gradient $\nabla \hat{u}(x)$ are (Hölder) continuous in a neighbourhood of $C$. It turns out that one of these hypotheses (based on certain ballance between external normals to the sets $C$ and $F$ ) can be adapted to the case of a Lipschitz continuous perturbation $\theta(\cdot)$.

We start in Section 2 with the basic definitions and an auxiliary statement. Then, in Section 3, we study the mathematical programming problem (7) from the viewpoint of the existence, uniqueness and the (Lipschitz) regularity of minimizers near the set $C$. The geometric condition ensuring such well-posedness is emphasized here. Finally, in Section 4 we examine the (Fréchet) differentiability of the value function $\hat{u}(\cdot)$ and justify the (Hölder) continuity of its gradient also under the assumption that either $F^{0}$ or the restriction $\left.\theta\right|_{C}$ is smooth.

\section{Preliminaries}

Given a convex closed bounded set $F \subset H$ with $0 \in \operatorname{int} F$ we consider the so called duality mapping $\mathfrak{J}_{F}: \partial F^{0} \rightarrow \partial F$, which associates to each $\xi^{*} \in \partial F^{0}$ the set of (normalized) linear functionals that support $F^{0}$ at $\xi^{*}$,

$$
\mathfrak{J}_{F}\left(\xi^{*}\right):=\left\{\xi \in \partial F:\left\langle\xi, \xi^{*}\right\rangle=1\right\} .
$$

In other words, $\mathfrak{J}_{F}\left(\xi^{*}\right)=\mathbf{N}_{F^{0}}\left(\xi^{*}\right) \cap \partial F$ where $\mathbf{N}_{F^{0}}\left(\xi^{*}\right)$ is the normal cone to the polar $F^{0}$ at $\xi^{*}$. It can be interpreted also as the subdifferential $\partial \rho_{F^{0}}\left(\xi^{*}\right)$ in the sense of Convex Analysis. For each dual pair $\left(\xi, \xi^{*}\right)$, i.e., such that $\xi \in \partial F$, $\xi^{*} \in \partial F^{0}$ and $\left\langle\xi, \xi^{*}\right\rangle=1$ let us define the modulus of rotundity (see [13])

$$
\widehat{\mathfrak{C}}_{F}\left(r, \xi, \xi^{*}\right):=\inf \left\{\left\langle\xi-\eta, \xi^{*}\right\rangle: \eta \in F,\|\xi-\eta\| \geq r\right\}, \quad r>0 .
$$

If the set $F$ is strictly convex (rotund) at $\xi$ w.r.t. $\xi^{*}$, i.e., $\widehat{\mathfrak{C}}_{F}\left(r, \xi, \xi^{*}\right)>0 \forall r>0$ then $\xi$ is an exposed point of $F$ and, in particular, $\xi$ is the unique element of 
$\mathfrak{J}_{F}\left(\xi^{*}\right)$. So, in this case $\xi$ is well defined whenever $\xi^{*}$ is fixed. Furthermore, given a set $U \subset \partial F^{0}$ we say that $F$ is uniformly rotund w.r.t. $U$ if

$$
\inf \left\{\widehat{\mathfrak{C}}_{F}\left(r, \xi, \xi^{*}\right): \xi^{*} \in U\right\}>0 \quad \forall r>0
$$

By [14, Proposition 2.1] this property is equivalent to the uniform continuity of $\mathfrak{J}_{F}(\cdot)$ in the following sense

$$
\sup _{\eta \in \mathfrak{J}_{F}\left(\eta^{*}\right)}\left\|\mathfrak{J}_{F}\left(\xi^{*}\right)-\eta\right\| \rightarrow 0 \quad \text { as } \quad\left\|\xi^{*}-\eta^{*}\right\| \rightarrow 0, \quad \xi^{*} \in U, \quad \eta^{*} \in \partial F^{0}
$$

(we clearly identify $\mathfrak{J}_{F}\left(\xi^{*}\right)$ with its element whenever it is a singleton). Uniform rotundity implies also the existence and the uniform continuity on $U$ of the Fréchet gradient $\nabla \rho_{F^{0}}\left(\xi^{*}\right)$.

Besides the concepts of Convex Analysis above we will use the following notations. For a lower semicontinuous function $\varphi: H \rightarrow \mathbb{R} \cup\{+\infty\}$ we denote by $\partial^{p} \varphi(x), \partial^{l} \varphi(x), \partial^{-} \varphi(x)$ and $\partial^{c} \varphi(x)$ the proximal, limiting (Mordukhovich), Fréchet and Clarke subdifferential, respectively, at a point $x, \varphi(x)<+\infty$. All the definitions and the basic facts of the calculus for non convex sets can be found, e.g., in [5]. Here we observe only that the inclusions

$$
\partial^{p} \varphi(x) \subset \partial^{-} \varphi(x) \subset \partial^{l} \varphi(x) \subset \partial^{c} \varphi(x)
$$

always hold, while one of the reverse inclusions takes place whenever $\varphi(\cdot)$ is regular at $x$ in some sense. For instance, $\varphi(\cdot)$ is said to be proximal (Clarke) regular at $x$ if $\partial^{p} \varphi(x)=\partial^{l} \varphi(x)$ (respectively, $\partial^{-} \varphi(x)=\partial^{c} \varphi(x)$ ).

If $C \subset H$ is a nonempty closed set then the notion of some kind of normal cone to $C$ at a point $x \in C$ can be given as the respective subdifferential of the indicator function $\mathbf{I}_{C}(\cdot)$ equal to 0 on $C$ and to $+\infty$ elsewhere. In particular, the proximal normal cone $\mathbf{N}_{C}^{p}(x):=\partial^{p} \mathbf{I}_{C}(x)$. Further on we denote by $\partial^{*} C:=$ $\left\{x \in \partial C: \mathbf{N}_{C}^{p}(x) \neq\{0\}\right\}$ the effective boundary, which is dense in $\partial C$.

Returning to the problem of minimization in (7) let us formulate first an approximation result, which is crucial for what follows. It can be proved similarly as Lemma 5.1 [13] by using the Ekeland's variational principle as well as the fuzzy sum rule for the proximal subdifferentials (see [5, Theorem 1.8.3]).

Lemma 1. Let $C \subset H$ be a nonempty closed set, and $\theta: H \rightarrow \mathbb{R} \cup\{+\infty\}$ be a lower semicontinuous function, lipschitzean on $C$. If $x \in H \backslash C$ and $\left\{x_{n}\right\} \subset C$ is a minimizing sequence for the function $y \mapsto \rho_{F^{0}}(x-y)+\theta(y)$ on $C$ then there exist another minimizing sequence $\left\{x_{n}^{\prime}\right\} \subset C$ and sequences $\left\{x_{n}^{\prime \prime}\right\},\left\{v_{n}\right\}$ and $\left\{\xi_{n}\right\}$ such that $v_{n} \in \partial^{p}\left(\left.\theta\right|_{C}\right)\left(x_{n}^{\prime}\right), \xi_{n} \in \partial \rho_{F^{0}}\left(x-x_{n}^{\prime \prime}\right)$ and $\left\|x_{n}^{\prime}-x_{n}\right\|+\left\|x_{n}^{\prime \prime}-x_{n}\right\| \rightarrow$ $0,\left\|v_{n}-\xi_{n}\right\| \rightarrow 0$ as $n \rightarrow \infty$. Here $\left.\theta\right|_{C}:=\theta+\mathbf{I}_{C}$.

Notice that if the points $x_{n}^{\prime}$ are such that $\partial^{p}\left(\left.\theta\right|_{C}\right)\left(x_{n}^{\prime}\right)=\partial^{p} \theta\left(x_{n}^{\prime}\right)+\mathbf{N}_{C}^{p}\left(x_{n}^{\prime}\right)$ and $\partial^{p} \theta\left(x_{n}^{\prime}\right) \subset \gamma F$ for some $0<\gamma<1$ then without loss of generality we can assume that $x_{n}^{\prime} \in \partial^{*} C$ and $v_{n} \in \partial F$. 


\section{Existence, Uniqueness and Regularity of Minimizers}

Our standing hypothesis in what follows will be a slightly strengthened condition than (9):

(H) there exists $0<\gamma<\frac{1}{\|F\|\left\|F^{0}\right\|}$ such that

$$
\theta(x)-\theta(y) \leq \gamma \rho_{F^{0}}(x-y) \quad \forall x, y \in C,
$$

where $\|F\|:=\sup \{\|\xi\|: \xi \in F\}$.

Hence $\theta(\cdot)$ is lipschitzean on $C$ with the constant $\gamma\|F\|$.

Given now an arbitrary point $x_{0} \in \partial C$ let us emphasize the main local assumptions, under which the well-posedness results hold:

$\left(\mathbf{H}_{1}\right)$ the mapping $x \mapsto \mathfrak{J}_{F^{0}}\left(\partial^{p}\left(\left.\theta\right|_{C}\right)(x) \cap \partial F\right)$ is single-valued and lipschitzean (with a constant $L=L\left(x_{0}\right)>0$ ) on the set

$$
C_{\delta}\left(x_{0}\right):=\left\{x \in \partial^{*} C:\left\|x-x_{0}\right\| \leq \delta\right\}, \delta>0
$$

$\left(\mathbf{H}_{2}\right)$ in the $\delta$-neighbourhood of $x_{0}$ the sum rule $\partial^{p}\left(\left.\theta\right|_{C}\right)(x)=\partial^{p} \theta(x)+$ $\mathbf{N}_{C}^{p}(x)$ takes place;

$\left(\mathbf{H}_{3}\right) F^{0}$ is uniformly rotund w.r.t. the set

$$
U_{\delta}\left(x_{0}\right):=\partial F \cap \bigcup_{x \in C_{\delta}\left(x_{0}\right)} \partial^{p}\left(\left.\theta\right|_{C}\right)(x) .
$$

For each $x \in H$ we denote by $\pi_{C}^{F, \theta}(x)$ the (possibly empty) set of all minimizers of the function $y \mapsto \rho_{F^{0}}(x-y)+\theta(y)$ on $C$.

Theorem 1. Under the hypotheses $\left(\mathbf{H}_{1}\right)-\left(\mathbf{H}_{3}\right)$ there exists a neighbourhood $\mathcal{U}\left(x_{0}\right)$ such that the mapping $x \mapsto \pi_{C}^{F, \theta}(x)$ is single-valued and continuous on $\mathcal{U}\left(x_{0}\right)$.

Proof. Let us give a sketch of the proof. Taking without loss of generality $\delta>0$ such that $\delta \gamma\|F\|<\left(1-\gamma\|F\|\left\|F^{0}\right\|\right) / L$, let us set

$$
\begin{aligned}
& \mathcal{U}\left(x_{0}\right):=\left\{x \in H:\left\|x-x_{0}\right\|<\frac{\left(1-\gamma\|F\|\left\|F^{0}\right\|\right) \delta}{2\|F\|\left\|F^{0}\right\|},\right. \\
& \left.\hat{u}(x)<\hat{u}\left(x_{0}\right)+\frac{1-\gamma\|F\|\left\|F^{0}\right\|}{L}-\delta \gamma\|F\|\right\} .
\end{aligned}
$$

Fix $x \in \mathcal{U}\left(x_{0}\right) \backslash C$ and a minimizing sequence $\left\{x_{n}\right\} \subset C$ of $y \mapsto \rho_{F^{0}}(x-y)+$ $\theta(y)$. Let us choose $\left\{x_{n}^{\prime}\right\} \subset C,\left\{x_{n}^{\prime \prime}\right\} \subset H, v_{n} \in \partial^{p}\left(\left.\theta\right|_{C}\right)\left(x_{n}^{\prime}\right)$ and $\xi_{n} \in$ $\partial \rho_{F^{0}}\left(x-x_{n}^{\prime \prime}\right)$ as in Lemma 1. Our goal is to prove that $\left\{x_{n}^{\prime}\right\}$ (hence $\left\{x_{n}\right\}$ as well) is a Cauchy sequence. 
To this end we show, first, that $\left\|x_{n}^{\prime}-x_{0}\right\| \leq \delta$. It follows then from $\left(\mathbf{H}_{2}\right)$ and from the remark after Lemma 1 that $x_{n}^{\prime} \in C_{\delta}\left(x_{0}\right)$ and $v_{n} \in \partial F$ for all $n$ large enough. Consider a (nonincreasing) sequence $\nu_{n} \rightarrow 0+$ such that

$$
\begin{aligned}
\left\|x_{n}^{\prime}-x_{n}\right\|+\left\|x_{n}^{\prime \prime}-x_{n}\right\| & \leq \nu_{n} \\
\rho_{F^{0}}\left(x-x_{n}^{\prime}\right)+\theta\left(x_{n}^{\prime}\right) & \leq \hat{u}(x)+\nu_{n} ; \\
\left\|v_{n}-\xi_{n}\right\| & \leq \nu_{n},
\end{aligned}
$$

$n=1,2, \ldots$ (see Lemma 1$)$. Then using the hypothesis $\left(\mathbf{H}_{3}\right)$ together with the property (10) (applied to the gauge $F^{0}$ ) gives that

$\beta_{n}:=\sup _{\|\xi-\eta\| \leq \nu_{n}} \sup \left\{\left\|\mathfrak{J}_{F^{0}}(\xi)-\mathfrak{J}_{F^{0}}(\eta)\right\|: \xi \in \partial F, \eta \in U_{\delta}\left(x_{0}\right)\right\} \rightarrow 0$ as $n \rightarrow \infty$.

Taking into account that $v_{n} \in U_{\delta}\left(x_{0}\right)$ (see (12)) and $\xi_{n} \in \partial F$ we obtain $\left\|\mathfrak{J}_{F^{0}}\left(\xi_{n}\right)-\mathfrak{J}_{F^{0}}\left(v_{n}\right)\right\| \leq \beta_{n}$, and, consequently, by $\left(\mathbf{H}_{1}\right)$

$$
\left\|\mathfrak{J}_{F^{0}}\left(\xi_{n}\right)-\mathfrak{J}_{F^{0}}\left(\xi_{m}\right)\right\| \leq 2 \beta_{n}+L\left\|x_{n}^{\prime}-x_{m}^{\prime}\right\|
$$

for all $m \geq n \geq 1$.

On the other hand, by the elementary properties of the convex subdifferentials and duality mappings we find that $\left(x-x_{n}^{\prime \prime}\right) / \rho_{F^{0}}\left(x-x_{n}^{\prime \prime}\right)=\mathfrak{J}_{F^{0}}\left(\xi_{n}\right)$, and hence

$$
\begin{aligned}
\left\|x_{n}^{\prime \prime}-x_{m}^{\prime \prime}\right\|= & \left\|\rho_{F^{0}}\left(x-x_{n}^{\prime \prime}\right) \mathfrak{J}_{F^{0}}\left(\xi_{n}\right)-\rho_{F^{0}}\left(x-x_{m}^{\prime \prime}\right) \mathfrak{J}_{F^{0}}\left(\xi_{m}\right)\right\| \leq \\
\leq & \rho_{F^{0}}\left(x-x_{n}^{\prime \prime}\right)\left\|\mathfrak{J}_{F^{0}}\left(\xi_{n}\right)-\mathfrak{J}_{F^{0}}\left(\xi_{m}\right)\right\|+ \\
& +\left|\rho_{F^{0}}\left(x-x_{n}^{\prime \prime}\right)-\rho_{F^{0}}\left(x-x_{m}^{\prime \prime}\right)\right|\left\|F^{0}\right\| .
\end{aligned}
$$

The terms $\left|\rho_{F^{0}}\left(x-x_{n}^{\prime \prime}\right)-\rho_{F^{0}}\left(x-x_{m}^{\prime \prime}\right)\right|$ and $\rho_{F^{0}}\left(x-x_{n}^{\prime \prime}\right)$ can be approximately estimated by $\left\|x_{n}^{\prime}-x_{m}^{\prime}\right\|$ and by $\hat{u}(x)-\hat{u}\left(x_{0}\right)$, respectively (we use for that the inequalities (14) and (15)). Hence, taking into account also (16) we deduce from (17) that

$$
\begin{aligned}
& \left\|x_{n}^{\prime}-x_{m}^{\prime}\right\| \leq\left\|x_{n}^{\prime \prime}-x_{m}^{\prime \prime}\right\|+2 \nu_{n} \leq \mu_{n}+ \\
& +\left[L\left(\mu_{n}^{\prime}+\hat{u}(x)-\hat{u}\left(x_{0}\right)+\delta \gamma\|F\|\right)+\gamma\|F\|\left\|F^{0}\right\|\right]\left\|x_{n}^{\prime}-x_{m}^{\prime}\right\|
\end{aligned}
$$

for all $m \geq n \geq 1$, where $\left\{\mu_{n}\right\}$ and $\left\{\mu_{n}^{\prime}\right\}$ are some sequences, converging to zero. We conclude the proof recalling the definition of the neighbourhood $\mathcal{U}\left(x_{0}\right)$. Thus, the limit $\bar{x}:=\lim _{n \rightarrow \infty} x_{n}^{\prime}$ will be (unique) minimizer from $\pi_{C}^{F, \theta}(x)$. The continuous dependence of this singleton on $x \in \mathcal{U}\left(x_{0}\right)$ also follows.

Remark 1. In fact, adapting the proof of Theorem 3.1 [14] we can show that the mapping $x \mapsto \pi_{C}^{F, \theta}(x)$ is locally lipschitzean on the same neighbourhood (13) with the Lipschitz constant tending to $+\infty$ as $x$ tends to the boundary $\partial \mathcal{U}\left(x_{0}\right)$.

\section{Differentiability of the Viscosity Solution}

We announce, first, a result on subdifferential regularity of the function (77) at a fixed point $x \notin C$, similar to Proposition 5.1 [14]. We see that the regularity relies upon well-posedness of the minimizers studied in the previous section. 
Theorem 2. Let us fix $x \in H \backslash C$ and assume that

$-\pi_{C}^{F, \theta}(y)$ is a singleton for each $y,\|x-y\| \leq \delta, \delta>0$;

- the restriction $\left.\theta\right|_{C}$ is proximally regular at $\bar{x}:=\pi_{C}^{F, \theta}(x)$;

- the following "centred" Hölder property

$$
\left\|\pi_{C}^{F, \theta}(x)-\pi_{C}^{F, \theta}(y)\right\| \leq K\|x-y\|^{\beta} \quad \forall y,\|x-y\| \leq \delta,
$$

holds with an exponent $1 / 2<\beta \leq 1$ and a constant $K=K(x)>0$.

Then the function $\hat{u}(\cdot)$ is Clarke regular at $x$. More precisely,

$$
\partial^{c} \hat{u}(x)=\partial^{-} \hat{u}(x)=\partial \rho_{F^{0}}(x-\bar{x}) \cap \partial^{-}\left(\left.\theta\right|_{C}\right)(\bar{x}) \neq \emptyset .
$$

Recalling Theorem 1 and Remark 1 we immediately obtain from the statement above that under the hypotheses $\left(\mathbf{H}_{1}\right)-\left(\mathbf{H}_{3}\right)$ the viscosity solution $\hat{u}(\cdot)$ is Clarke regular and (18) holds for all $x \in \mathcal{U}\left(x_{0}\right)$ where the neighbourhood $\mathcal{U}\left(x_{0}\right)$ is defined by (13). Thus, for the (Fréchet) continuous differentiability it suffices to require that the intersection $\partial \rho_{F^{0}}(x-\bar{x}) \cap \partial^{-}\left(\left.\theta\right|_{C}\right)(\bar{x})$ is a singleton continuously depending on $x \in \mathcal{U}\left(x_{0}\right)$. However, this is difficult to verify directly because the mapping (18) (which is nothing else than the Fréchet gradient $\nabla \hat{u}(x)$ ) depends on the point $x$ through a priori unknown function $\pi_{C}^{F, \theta}(\cdot)$. On the other hand, this condition splits into two different hypotheses regarding the smoothness either of both the function $\theta(\cdot)$ and the set $C$, or of the polar gauge $F^{0}$. Moreover, such hypotheses can be given plainly in terms of boundary points of the sets $C$ and $F^{0}$. Notice that $C$ is said to be with smooth boundary near $x_{0}$ if $\mathbf{N}_{C}^{l}(x) \cap \partial \bar{B}$ is a singleton (say $\left\{\mathfrak{n}_{C}(x)\right\}$ ) continuously depending on $x \in \partial C$, $\left\|x-x_{0}\right\| \leq \delta, \delta>0$. Also the smoothness of $F^{0}$ can be equivalently substituted by the rotundity assumption for $F$.

Thus, we arrive at the following result.

Theorem 3. Given $x_{0} \in \partial C$ and $\delta>0$ let us assume the hypotheses $\left(\mathbf{H}_{1}\right)-$ $\left(\mathbf{H}_{3}\right)$. Suppose also that at least one of the following two conditions holds:

(i) $C$ has smooth boundary, and $\theta(\cdot)$ is of class $\mathcal{C}^{1}$ near $x_{0}$;

(ii) $F$ is rotund w.r.t. each $\xi^{*} \in \mathfrak{J}_{F^{0}}\left(\partial^{p}\left(\left.\theta\right|_{C}\right)(x)\right), x \in \partial C$ with $\left\|x-x_{0}\right\| \leq \delta$.

Then $\hat{u}(\cdot)$ is Fréchet continuously differentiable on a neighbourhood $\mathcal{U}\left(x_{0}\right)$. Furthermore, in the first case we have

$$
\nabla \hat{u}(x)=\nabla \theta(\bar{x})+\lambda \mathfrak{n}_{C}(\bar{x}),
$$

where $\lambda=\lambda(\bar{x})>0$ is the unique positive root of the equation

$$
\rho_{F}\left(\nabla \theta(\bar{x})+\lambda \mathfrak{n}_{C}(\bar{x})\right)=1,
$$

while in the second

$$
\nabla \hat{u}(x)=\nabla \rho_{F^{0}}(x-\bar{x}) .
$$

Here $\bar{x}:=\pi_{C}^{F, \theta}(x), x \in \mathcal{U}\left(x_{0}\right)$, as before. 
Proof. If the condition (i) is fulfilled then taking into account that $\pi_{C}^{F, \theta}(\cdot)$ is single-valued and continuous on $\mathcal{U}\left(x_{0}\right)$, and that

$$
\partial^{l}\left(\left.\theta\right|_{C}\right)(\bar{x})=\nabla \theta(\bar{x})+\mathbf{N}_{C}^{l}(\bar{x})=\left\{\nabla \theta(\bar{x})+\lambda \mathfrak{n}_{C}(\bar{x}): \lambda \geq 0\right\}
$$

whenever $x \in \mathcal{U}\left(x_{0}\right)$, we obtain that the intersection in (18) reduces to the singleton $\left\{\nabla \theta(\bar{x})+\lambda(\bar{x}) \mathfrak{n}_{C}(\bar{x})\right\}$ (see also (11)). The continuity of $\nabla \hat{u}(\cdot)$ can be shown now by the standart implicit function argument.

Under the alternative assumption (ii) it sufices to observe that due to a necessary condition of optimality (in the proximal form) the (unique) minimizer $\bar{x}=\pi_{C}^{F, \theta}(x)$ must satisfy the relationship

$$
\partial^{p}\left(\left.\theta\right|_{C}\right)(\bar{x}) \cap \mathbf{N}_{F^{0}}\left(\frac{x-\bar{x}}{\rho_{F^{0}}(x-\bar{x})}\right) \cap \partial F \neq \emptyset .
$$

Then, it follows from (19) that $\frac{x-\bar{x}}{\rho_{F^{0}}(x-\bar{x})} \in \mathfrak{J}_{F^{0}}(\xi)$ for some $\xi \in \partial^{p}\left(\left.\theta\right|_{C}\right)(\bar{x})$. Therefore, if $x \in \mathcal{U}\left(x_{0}\right)$ then $\bar{x}$ is closed to $x_{0}$ as well, and taking $\xi^{*}=\frac{x-\bar{x}}{\rho_{F^{0}}(x-\bar{x})}$ we deduce from (ii) that $\rho_{F^{0}}(\cdot)$ is (Fréchet) continuously differentiable at $\xi^{*}$. So, the intersection in (18) reduces to $\left\{\nabla \rho_{F^{0}}(x-\bar{x})\right\}$, and the continuity w.r.t. $x$ also follows.

Remark 2. If in addition to the hypothesis (i) in Theorem 3 we assume that both unit normal vector $\mathfrak{n}_{C}(\cdot)$ and the gradient $\nabla \theta(\cdot)$ are Hölder continuous with an exponent $0<\alpha \leq 1$ on a $\delta$-neighbourhood of $x_{0}$ then $\nabla \hat{u}(\cdot)$ will be also Hölder continuous near $x_{0}$ with the same exponent (we say that $\hat{u}(\cdot)$ is of class $\mathcal{C}_{\text {loc }}^{1, \alpha}$ on $\left.\mathcal{U}\left(x_{0}\right)\right)$. One can derive the Hölder inequality for $\nabla \hat{u}(\cdot)$ by using Theorem 3 and the estimates for the Hausdorff distance between the polars for convex solids (see Lemma 2 [1] ).

\section{References}

[1] Bardi, M., Capuzzo-Dolcetta, I.: Optimal Control and Viscosity Solutions of Hamilton-Jacobi-Bellman Equations. Birkhäuser, Boston (1997)

[2] Barles, G.: Solutions de Viscosité des Équations de Hamilton-Jacobi. Springer, Berlin (1994)

[3] Bressan, A.: Hamilton-Jacobi equations and optimal control. An illustrated tutorial. Trondheim, NTNU (2001)

[4] Cardaliaguet, P., Dacorogna, B., Gangbo, W., Georgy, N.: Geometric restrictions for the existence of viscosity solutions. Ann. Inst. Henri Poincaré 16, 189-220 (1999)

[5] Clarke, F.H., Ledyaev, Y.S., Stern, R.J., Wolenski, P.R.: Nonsmooth Analysis and Control Theory. Springer, New York (1998)

[6] Crandall, M., Lions, P.-L.: Viscosity solutions of Hamilton-Jacobi equations. Trans. Amer. Math. Soc. 277, 1-42 (1983)

[7] Crandall, M., Lions, P.-L.: Hamilton-Jacobi equations in infinite dimensions. I: Uniqueness of viscosity solutions. J. Funct. Anal. 62, 379-396 (1985)

[8] Crandall, M., Lions, P.-L.: Hamilton-Jacobi equations in infinite dimensions. II: Existence of viscosity solutions. J. Funct. Anal. 65, 368-405 (1986) 
[9] Colombo, G., Thibault, L.: Prox-regular sets and applications. In: Gao, D.Y., Motreano, D. (eds.) Handbook on Nonconvex Analysis. International Press, Boston (2010)

[10] Colombo, G., Wolenski, P.R.: Variational Analysis for a class of minimal time functions in Hilbert spaces. J. Convex Anal. 11, 335-361 (2004)

[11] Dal Maso, G., Goncharov, V.V., Ornelas, A.: A Lipschitz selection from the set of minimizers of a nonconvex functional of the gradient. Nonlin. Anal.: Theory, Meth. and Appl. 37, 707-717 (1999)

[12] Douglis, A.: The continuous dependence of generalized solutions of non-linear partial differential equations upon initial data. Comm. Pure Appl. Math. 14, 267284 (1961)

[13] Goncharov, V.V., Pereira, F.F.: Neighbourhood retractions of nonconvex sets in a Hilbert space via sublinear functionals. J. Convex Anal. 18, 1-36 (2011)

[14] Goncharov, V.V., Pereira, F.F.: Geometric conditions for regularity in a timeminimum problem with constant dynamics. J. Convex Anal. 19, 631-669 (2012)

[15] Kružkov, S.N.: Generalized solutions of the Hamilton-Jacobi equation of the Eikonal type. I. Math. USSR Sbornik 27, 406-446 (1975)

[16] Oleinik, O.A.: On discontinuous solutions of non-linear differential equations. Uspehi Mat. Nauk 12, 3-73 (1957) 